\title{
DOENÇA DE CROHN E GESTAÇÃO
}

\author{
CROHN'S DISEASE AND PREGNANCY \\ Alessandra Cristina Marcolin ${ }^{1}$; Carla Vitola Gonçalves ${ }^{1}$; Cleusa Cascaes Dias²; \\ Luiz Ernesto de Almeida Troncon ${ }^{3} \&$ Geraldo Duarte ${ }^{4}$

\begin{abstract}
${ }^{1}$ Aluna do curso de pós-graduação, no Setor de Patologia Obstétrica; ${ }^{2}$ Médica Contratada. Departamento de Ginecologia e Obstetrícia. ${ }^{3}$ Docente Associado. Departamento de Clínica Médica. ${ }^{4}$ Docente Associado. Departamento de Ginecologia e Obstetrícia. Faculdade de Medicina de Ribeirão Preto-USP.

CorRespondênCIA: Geraldo Duarte - Avenida Bandeirantes, 3900 - Hospital das Clínicas da Faculdade de Medicina de Ribeirão Preto - USP, Ribeirão Preto, São Paulo - 14049-900 - Fone: (016) 602-2588 - e-mail:_gduarte@fmrp.usp.br
\end{abstract}

MARCOLIN AC; GONÇALVES CV; DIAS CC; TRONCON LEA \& DUARTE G. Doença de Crohn e gestação. Medicina, Ribeirão Preto, 34: 64-69, jan./mar.2001.

RESUMO: A doença de Crohn é uma enterite granulomatosa, transmural, crônica, de etiologia desconhecida que pode acometer qualquer parte do trato gastrintestinal, atingindo, preferencialmente, o íleo distal, os cólons e a região anorretal. Seu curso é variável, podendo ser de caráter indolente e prolongado, seguindo um padrão de exacerbações e remissões. A incidência da doença de Crohn aumentou nos últimos 30 anos e, na maioria dos casos, as manifestações clínicas iniciam-se na segunda e terceira décadas da vida, acometendo as mulheres em pleno período reprodutivo. O fator mais importante para o prognóstico gestacional, nessas mulheres, é a atividade da doença no início da gravidez. Complicações obstétricas como aborto espontâneo, parto pré-termo, malformações fetais e retardo de crescimento intra-útero, ocorrem com as recidivas da doença no período periconcepcional ou durante o primeiro trimestre gestacional. $A$ evolução da doença de Crohn, durante a gestação, também dependerá da atividade da mesma, no início da gestação.

Nesta revisão, os autores avaliaram os principais aspectos etiológicos, clínicos e terapêuticos da doença de Crohn durante a gravidez, discutindo detalhadamente os efeitos da doença sobre a gestação e as conseqüências da gravidez sobre a evolução dessa doença.

UNITERMOS: Doença de Crohn. Gravidez. Fertilidade.

\section{INTRODUÇAO}

A doença de Crohn foi descrita, pela primeira vez, por Crohn et al., em 1932, sendo designada como "ileíte regional". Estudos posteriores possibilitaram entendê-la como uma enterite granulomatosa, transmural, crônica e de etiologia desconhecida ${ }^{(1)}$. Observa-se que essa enfermidade pode acometer qualquer parte do trato gastrintestinal, mas atinge, preferencialmente, o íleo distal, os cólons e a região anorretal. $\mathrm{O}$ acometimento da serosa, do mesentério, dos linfonodos regionais e de outros órgãos ocorre com menor freqüência ${ }^{(2)}$.

Um dos aspectos característicos da doença de Crohn é a demarcação nítida do acometimento segmentar do intestino, de modo que segmentos enfermos são separados por intestino normal. Na fase inicial, ocorre um espessamento globoso, edematoso e hiperêmico da mucosa intestinal, resultando em minúsculas ulcerações. Nos casos crônicos, úlceras e fissuras podem penetrar profundamente e formar trajetos fistulosos com outras alças do intestino, aumentando a 
ocorrência de abscessos intracavitários ${ }^{(1)}$. Histologicamente, podem ser observados granulomas não caseosos e importante reação inflamatória crônica inespecífica, com o peculiar acometimento transmural ${ }^{(2)}$.

O diagnóstico diferencial da doença de Crohn com a retocolite ulcerativa, que acomete predominantemente os cólons, nem sempre pode ser estabelecido, principalmente no caso de gestantes, com quem os procedimentos diagnósticos são limitados ${ }^{(3)}$.

O curso da doença de Crohn é variável, podendo ser de caráter indolente e prolongado. Habitualmente, a evolução segue um padrão de exacerbações e remissões, com tendência à recidiva, apesar do tratamento clínico intensivo ou após cirurgia. Essa enfermidade associa-se a um custo significativo e freqüentemente impõe alterações substanciais no estilo de vida do paciente.

Sua incidência parece estar aumentando em todo o mundo, nos últimos 30 anos. A doença apresenta distribuição mundial, tem ocorrência igual em ambos os sexos, parece ser mais comum em brancos, em judeus e entre parentes próximos de pessoas acometidas. Na maioria absoluta dos casos, as manifestações clínicas iniciam-se na segunda e terceira décadas da vida. Deste modo, a doença de Crohn acomete as mulheres em pleno período reprodutivo ${ }^{3}$. Esse dado epidemiológico justifica a preocupação do tocoginecologista com a doença, pois, além de apresentar influências negativas sobre a fertilidade, representa risco adicional para a saúde materna e fetal.

\section{ETIOLOGIA}

Até o momento,não foi estabelecido um fator etiológico para a doença de Crohn. Os padrões de prevalência dessa condição sugerem a importância tanto de fatores ligados ao hospedeiro, quanto aqueles de ordem ambiental. Algumas teorias sobre a causa dessa doença são resumidas a seguir.

1) Fatores infecciosos: os agentes mais freqüentemente arrolados na gênese da doença de Crohn são os vírus, dentre eles os rotavírus, o vírus de EpsteinBarr e alguns RNA vírus citopáticos. Bactérias semelhantes a Pseudomonas, anaeróbios entéricos, Mycobacterium Kansaii com parede celular defeituosa e Yersinia enterocolitica também foram implicados na causa da doença, porém essa associação ainda carece de provas definitivas ${ }^{(2)}$.

2) Fatores imunológicos: evidências apontam para os distúrbios imunológicos como os princi- pais causadores da doença de Crohn. Observam-se alterações da imunidade humoral e celular, como secreção aumentada de imunoglobulinas de mucosa, anormalidades nos números de linfócitos $\mathrm{B}$ e $\mathrm{T}$ circulantes e respostas exacerbadas de macrófagos. Parece que complexos antigenoanticorpos, circulantes estão envolvidos na causalidade da doença extra-intestinal ${ }^{(1)}$. Mas, ainda há dúvidas se esses fenômenos imunológicos são causais ou secundários ao processo patológico ${ }^{(2)}$.

3) Fatores psicogênicos: sabe-se que o sistema nervoso pode influenciar profundamente as funções motoras, secretoras, vasculares e metabólicas do sistema digestivo, levando à exacerbação da doença intestinal ${ }^{(1)}$.

\section{QUADRO CLÍNICO}

O quadro clínico da doença de Crohn é muito variável e depende da extensão e do segmento intestinal envolvido. Quando há envolvimento do íleo, encontramos a enterite regional "clássica" e o quadro clínico mais característico da doença. As manifestações clínicas são representadas por dor abdominal com piora no período pós-prandial. A dor pode se associar à febre baixa, emagrecimento e vários episódios diários de diarréia, sem sangue ${ }^{(1)}$. Os sintomas podem ocorrer com períodos de exacerbações e remissões. Quando há o comprometimento exclusivo do intestino grosso, os sintomas e sinais são semelhantes aos da retocolite ulcerativa, com predomínio de diarréia e sangramento pelo reto.

Uma característica freqüente da doença de Crohn é a formação de fístulas. Cerca de um terço das pacientes desenvolvem fístulas enterais, perirretais ou perineais, que se manifestam por dor e formação de massas abdominais ou abscessos. Em 1994, Wulfeck et al. descreveram um caso de fístula enteruterina, enfatizando as dificuldades diagnósticas e elevada morbimortalidade materna e perinatal ${ }^{(4)}$. Muitas vezes, as fístulas enterais podem cursar como abscessos tamponados $^{(5)}$ ou manifestarem-se por quadros extraintestinais, a exemplo dos abscessos hepáticos. A complicação pode cursar como um quadro septicêmico, de diagnóstico difícil, sobretudo durante a gestação ${ }^{(6)}$.

Manifestações extra-intestinais podem ocorrer, destacando-se as alterações articulares, como a espondilite anquilosante, as manifestações cutaneomucosas representadas pelas estomatites e outros quadros extra-intestinais menos freqüentes, como as afecções renais, biliares e oculares. Elas podem pre- 
ceder o diagnóstico da doença de Crohn intestinal ou podem ocorrer em qualquer momento da doença $a^{(1)}$.

Alguns autores já citaram acometimento vulvar pela doença de Crohn e apontaram as dificuldades de diagnóstico e de manejo nesses $\operatorname{casos}^{(7)}$, podendo haver até deiscência de episiorrafia em pacientes com a doença ${ }^{(8)}$.

\section{EFEITOS DA DOENÇA DE CROHN SOBRE A GRAVIDEZ}

Publicações das décadas de 1950 e de 1960 levaram à idéia de que a doença de Crohn apresenta efeitos adversos sobre a gestação. A origem de tal conceito decorreu do fato de que, naquela época, os corticóides e a sulfassalazina, bem como o tratamento cirúrgico, não eram utilizados tão amplamente como passaram a ser nas décadas seguintes. Estudos mais recentes têm demonstrado os reais efeitos da doença sobre o processo reprodutivo.

A fertilidade parece ser igual ${ }^{(9)}$ ou levemente reduzida nas pacientes portadoras da doença ${ }^{(10)}$. Essa diminuição poderia estar relacionada à eventual obstrução tubárea, à diminuição da libido, às deficiências nutricionais decorrentes da doença e, em especial, à própria atividade inflamatória da doença ativa ${ }^{(11)}$. Por outro lado, a fertilidade reduzida pode representar mais a escolha da paciente, refletindo a falta de desejo de engravidar devido à debilidade geral ${ }^{(12)}$. No entanto, infertilidade sem causa aparente é mais freqüente nas mulheres submetidas à cirurgia devido à doença de Crohn $^{(13)}$.

Evidências indicam que o fator mais importante para o prognóstico gestacional, em mulheres portadoras de doença de Crohn, é a atividade da doença no início da gravidez. Nas pacientes com doença em remissão, a frequiência de aborto espontâneo, de partos pré-termo, de malformações fetais e de recém-nascidos de baixo peso é semelhante àquela da população geral $^{(11)}$. Avaliando 177 gestantes com a doença de Crohn, Baird et al.,em 1990, concluíram que não houve maior risco de perdas gestacionais nessas pacientes $^{(12)}$. Em 1991, Schneider et al., estudando 107 gestantes portadoras da doença inflamatória intestinal, mostraram que a média de peso e de estatura de seus recém-nascidos não diferiram dos valores registrados para gestações anteriores à doença ${ }^{(10)}$. Mais recentemente, Korelitz, em 1998, também reforçou a ausência de maiores repercussões perinatais em gestantes com a doença de Crohn ${ }^{(14)}$.
Não obstante, a situação obstétrica, em portadoras da doença de Crohn pode se alterar nos casos em que a concepção ocorre na fase inicial da doença, ou coincide com exacerbações observadas ao longo do seu curso. Em 1990, Woolfson et al. estudaram 78 gestações em 50 pacientes com doença, observando que $27 \%$ delas apresentaram anormalidades, como aborto espontâneo, prematuridade, retardo de crescimento intra-útero e desconforto respiratório do recémnascido. Tais complicações ocorreram em $21 \%$ das pacientes com doença inativa, mas, em 50\% daquelas com doença ativa, mostrando a influência negativa da atividade da doença sobre o resultado perinatal ${ }^{(15)}$. Deve-se, portanto, enfatizar a importância de se planejar a gravidez para períodos de remissão da doença, dando assistência e orientando a paciente ${ }^{(9)}$.

Por outro lado, a literatura mostra que alguns autores ainda acreditam na maior incidência de repercussões perinatais em pacientes com a doença de Crohn. Em 1998, Larzilliere \& Beau e Fonager et al. apontaram ocorrência aumentada de recém-nascidos de baixo peso e de partos pré-termo, mesmo em períodos de efetivo controle da doença ${ }^{(16,17)}$.

Com relação ao parto, pesquisas mostram alta taxa de acometimento perineal pela doença de Crohn, ocorrendo após parto vaginal com episiotomia, mesmo em casos sem existência prévia de doença no lo$\mathrm{cal}^{(18)}$. Paralelamente, observou-se incidência aumentada de cesárea eletiva na população acometida pela doença $(20,9 \%)$, quando comparada com a da população geral (15\%), como exemplificado por Ilnyckyji et al., em 1999(19). Porém, Rogers \& Katz, em 1995, mostraram que a cesárea profilática não evita o acometimento perineal em pacientes sem tal acometimento prévio, o que os levou a sugerir que a cesárea deva ser reservada para as indicações obstétricas específicas $^{(20)}$.

De modo geral, as evidências disponíveis indicam que a doença de Crohn não constitui contra-indicação para a gravidez e nem para o parto, devendo o médico cuidar para que gravidez ocorra durante os períodos de remissão da doença.

\section{EFEITOS DA GRAVIDEZ SOBRE A DOEN- ÇA DE CROHN}

A idéia de que a gravidez apresenta efeitos adversos sobre a doença de Crohn não é verdadeira. A maioria das pacientes com doença inativa no momento da concepção tende a permanecer assintomática 
durante a gestação. Observa-se que menos de um terço dessas pacientes poderão reativar a doença neste período, o que ocorre, mais freqüentemente, no primeiro trimestre da gravidez ou no puerpério ${ }^{(11)}$. No entanto, essa taxa é semelhante à encontrada em mulheres não gestantes, portadoras da doença, observadas durante um período equivalente ao da gestação ${ }^{(21)}$.

Em 1990, Woolfson et al. demonstraram que $25 \%$ das gestantes com doença inativa tiveram recidivas, mas $44 \%$ daquelas com doença ativa apresentaram melhora do quadro ${ }^{(15)}$. Castiglione et al., em 1996, avaliaram 29 gestações, em 18 pacientes com doença de Crohn, observando 14\% de recidivas durante a gravidez e $17 \%$ no período puerperal. Durante os três anos que se seguiram à gestação, o número de recidivas/ano foi significativamente menor, quando comparado ao dos três anos que antecederam a gestação. Os autores atribuem o fato à melhora do estado nutricional nesse grupo de gestantes ${ }^{(22)}$. Além disso, várias outras séries têm evidenciado ausência de efeitos da gestação sobre a doença de $\mathrm{Crohn}^{(9,13)}$.

Em alguns casos, pode haver ligeira piora da doença durante a gravidez, caracterizando-se por episódios diarréicos, emagrecimento e ganho de peso inadequado durante a gestação ${ }^{(20)}$.

A variabilidade própria da doença não permite predizer a evolução da gestação em curso ${ }^{(11)}$. A tendência atual é acreditar que o curso da doença dependerá da atividade da mesma, não só antes, como também durante a concepção ${ }^{(14)}$.

\section{DIAGNÓSTICO}

O diagnóstico da doença de Crohn baseia-se na clínica e propedêutica complementar que envolve, principalmente, exames endoscópicos, radiológicos e ultra-sonográficos.

Apesar de a endoscopia pouco contribuir para o diagnóstico das alterações do intestino delgado, seu uso é preferencial na investigação das pacientes durante a gravidez, pois não representa risco para o feto. A colonoscopia é útil para avaliar a extensão e a intensidade do acometimento, além de permitir a realização de biópsias ${ }^{(11)}$. Porém, deve-se ter cautela com eventuais traumatismos da parede colônica, que podem ocorrer em estágios mais avançados da gestação ${ }^{(23)}$. A retossigmoidoscopia é bem tolerada e pode ser normal em mais de metade das pacientes ${ }^{(1,23)}$. A ressonância magnética também é um método diag- nóstico importante na gravidez por ser seguro e não invasivo $^{(24)}$.

A investigação radiológica deve ser realizada sempre que indicada, com algumas limitações. O exame radiológico apresenta riscos já conhecidos, quando realizado no primeiro trimestre gestacional, devendo ser evitado neste período ${ }^{(23)}$. Alterações características são melhor avaliadas com exames contrastados, utilizando bário, e incluem irregularidades do padrão mucoso, com áreas lesadas, separadas por áreas de aspecto normal, estreitamento segmentar e formação de fístula enteroentérica ${ }^{(1)}$.

A ecografia do abdome tem se mostrado útil no diagnóstico da doença de Crohn e de suas complicações, ocupando lugar de destaque durante a gestação, por ser um método inócuo e não invasivo. Apresenta boas taxas de sensibilidade e especificidade, com base na caracterização de segmentos intestinais com paredes espessadas pelo processo inflamatório ${ }^{(25)}$. Além disso, complicações, como abcessos decorrentes de fístulas e obstrução intestinal, podem também ser diagnosticadas $^{(26)}$.

Alterações laboratoriais na doença de Crohn incluem: anemia, leucocitose e aumento da velocidade de hemossedimentação, todos secundários à atividade inflamatória exacerbada, porém de valor limitado durante a gestação. Observamos, também, alterações eletrolíticas nos casos de diarréia importante e hipoalbuminemia ${ }^{(11)}$.

\section{TRATAMENTO}

O controle da doença de Crohn requer acompanhamento cuidadoso dos casos e tratamento clínico e/ou cirúrgico. Ressalta-se que a terapia, durante a gravidez, assemelha-se muito àquela dispensada a pacientes fora da gestação.

A paciente deve receber uma dieta adequada às suas necessidades nutricionais. Aquelas com acometimento ileal devem evitar o excesso das fibras pelo risco de obstrução nesse segmento. Nas pacientes com esteatorréia, a menor ingestão de gorduras melhora a diarréia ${ }^{(111)}$. Em 1991, Teahon et al. demonstraram que uma dieta suplementar adequada, pode ser uma forma segura de tratamento durante a gravidez, em casos sintomáticos ${ }^{(27)}$.

A gestante hospitalizada representa um problema terapêutico diferente. Uma conduta nesse grupo é fazer "repousar" o intestino afetado, removendo-se o 
estímulo da ingestão alimentar. Dietas enterais também podem ser benéficas. Em casos mais graves ou de fístulas, pode ser utilizado o suporte nutricional parenteral $^{(1,11)}$.

Com relação à terapia medicamentosa, o tratamento sintomático para a dor abdominal se faz por meio de analgésicos e antiespasmódicos. Não se utilizam antidiarréicos. As drogas mais freqüentemente utilizadas com finalidade antiinflamatória são os corticosteróides, a sulfassalazina e, ocasionalmente, os agentes imunossupressores.

Os corticóides são altamente eficazes no tratamento da fase aguda e de exacerbação da doença ${ }^{(28)}$. Podem ser utilizados durante a gravidez, com segurança, sem aumentar o risco de complicações perinatais $^{(15,29)}$. O principal inconveniente da corticoterapia é a incidência de efeitos colaterais, que pode ser minimizada com o advento de novos agentes, como a budesonida $^{(28)}$.

A sulfassalazina é composta por uma sulfonamida, a sulfapiridina, e um salicilato, o ácido 5-aminossalicílico (5-ASA). A droga tem eficácia comprovada na exacerbação da doença e seu efeito mais notável é a manutenção da remissão. É utilizada por via oral e metabolizada no intestino pelas bactérias, com liberação do 5-ASA, inibidor da síntese de mediadores da inflamação. Porém, possui efeitos colaterais relacionados à sulfapiridina e, quando utilizada, necessita de suplementação de ácido folínico ${ }^{(11)}$. Hoje, existem salicilatos revestidos, que resistem ao suco gástrico, como a mesalamina e a olsalazina, com taxa reduzida de efeitos colaterais. Habal et al., em 1993, não encontraram evidências clínicas ou da bioquímica neonatal indicativas de qualquer anormalidade em gestantes usuárias de 5-ASA por toda a gravidez ${ }^{(30)}$. Tennenbaum et al., em 1999, enfatizaram que não há necessidade de suspender o uso da droga durante o período gestacional ${ }^{(31)}$. A utilização dessas drogas durante a lactação parece ser segura ${ }^{(25)}$.

Imunossupressores, como a azatioprina e a 6mercaptopurina, têm sido utilizados com bons resultados no tratamento da doença de Crohn ${ }^{(1)}$. Têm indicação em casos que necessitam do emprego contínuo de corticosteróides ("corticodependência" ou "corticorresistência"), havendo um efeito "poupador" de corticóides, possibilitando o uso de doses menores de esteróides. Seu uso está associado a prejuízo da imunidade fetal, retardo de crescimento e prematuridade. Connell \& Miller em 1999, ressaltaram as controvérsias quanto ao uso de 6-mercaptopurina na gestação e a proibição da gravidez em usuárias de methotrexate, devido aos riscos de aborto e de malformações fetais $^{(29)}$.

Assim, parece que os efeitos da inflamação sobre o feto durante a fase ativa da doença são mais prejudiciais que o tratamento medicamentoso.

No que se refere ao tratamento cirúrgico, suas indicações não se modificam por causa da gravidez ${ }^{(32)}$.

MARCOLIN AC; GONÇALVES CV; DIAS CC; TRONCON LEA \& DUARTE G. Crohn's disease and pregnancy. Medicina, Ribeirão Preto, 34: 64-69, jan./march 2001.

ABSTRACT: Crohn's disease is a chronic transmural granulomatous enteritis of unknown etiology that may affect any part of the gastrointestinal tract, preferentially involving the distal ileum, the colons and the anorectal region. Its course is variable, of a dormant and prolonged nature, with a pattern of exacerbations and remissions. The incidence of Crohn's disease has greatly increased in the last 30 years, with the absolute majority of cases starting to show clinical manifestations in the second and third decades of life, and with women being affected during their reproductive years. The disease activity in the beginnig of pregnancy is the most important factor affecting both the prognosis of Crohn's disease and gestational prognosis. Obstetrical complications as spontaneous abortion, preterm delivery, fetal malformation and intrauterus growth retardation happen when the disease is active in the periconceptional period or during the first trimester of pregnancy.

In the present review, the authors evaluate the major etiological, clinical and therapeutic aspects of Crohn's disease during pregnancy, with a detailed discussion of the effects of the disease on pregnancy and the consequences of gestation for the evolution of the disease.

UNITERMS: Crohn Disease. Pregnancy. Fertility. 


\section{REFERÊNCIAS BIBLIOGRÁFICAS}

1 - HANAUER SB. Doença inflamatória intestinal. In: BENNETT JC \& PLUM F, eds. Cecil - Tratado de medicina interna, $20^{\text {th }}$ ed, Editora Guanabara Koogan , Rio de Janeiro, p. 782791, 1997.

2 - DAYAL Y \& DELELLIS RA. Trato gastrintestinal. In: COTRAN RS; KUMAR V \& ROBBINS SL, eds. Patologia estrutural e funcional, $4^{\text {th }}$ ed, Editora Guanabara Koogan, Rio de Janeiro, p.713 - 717, 1991.

3 - SCOTT LD. Gastrointestinal disease in pregnancy. In: CREASY RK \& RESNIK R, eds. Maternal-fetal medicine, $4^{\text {th }}$ ed, W. B. Saunders, Philadelphia, p. 1038-1056, 1999.

4 - WULFECK D; WILLIAMS T; AMIN A \& HUANG TY. Crohn's disease with unusual enterouterine fistula in pregnancy. $\mathbf{J}$ Ky Med Assoc 92: 267-269, 1994.

5 - SOLOMON MJ; DEVAAK; CORCORAN SJ \& GALLAGHER N Postpartum avulsion of the terminal ileal wall in Crohn's disease. Aust N Z J Surg 66: 849-851, 1996.

6 - DOMINGUEZS; BOUDGHENE F; HOURY S; HEIM N; CALLARD $P$ \& GRANGE JD. Septic shock during the immediate postpartum period revealing a liver abscess in a woman with Crohn's disease. Gastroenterol Clin Biol 23: 775-778,1999.

7 - BITRI M; CHELLY H; BEN OSMANE A \& HAOUET S. Crohn's disease with isolated vulvar localization and pregnancy. A case report. J Gynecol Obstet Biol Reprod (Paris) 23: 712-716,1994.

8 - MATORRAS R; GARCIAA; GUTIERREZDE TERAN G; IDIONDO V; MARTINEZ-BOURIO JL \& SANJURJO P. Crohn's disease presented by episiotomy dehiscence and perineal fistula. Zentralbl Gynakol 117: 491-493,1995.

9 - CLEANDRE D; DUFOUR P; TIBERGHIEN B; BERNARDI C; VINATIER D \& MONNIER JC. Chronic idiopathic enterocolitis and pregnancy. Reciprocal repercussions and treatment. J Gynecol Obstet Biol Reprod (Paris) 22: 59-65, 1993.

10 - SCHNEIDER W \& HANERT E. Pregnancy and chronic inflammatory bowel diseases. Z Gesamte Inn Med 46: 602-606, 1991.

11 - PEREIRAAS \& PEREIRA FILHO RA. Gastroenteropatias. In: NEME B, ed. Obstetrícia básica, $2^{\text {th }}$ ed, Editora Sarvier, São Paulo, p. 430 - 435, 2000.

12 - BAIRD DD; NARENDRANATHAN M \& SANDLER RS. Increased risk of preterm birth for women with inflammatory bowel disease. Gastroenterology 99: 987-994, 1990.

13 - HUDSON M; FLETT G; SINCLAIR TS; BRUNT PW; TEMPLETON A \& MOWAT NA. Fertility and pregnancy in inflammatory bowel disease. Int J Gynaecol Obstet 58: 229-237, 1997.

14 - KORELITZ BI. Imflammatory bowel disease and pregnancy. Gastroenterol Clin North Am 27: 213-224, 1998.

15 - WOOLFSON K; COHEN Z \& MCLEOD RS. Crohn's disease and pregnancy. Dis Colon Rectum 33: 869-873,1990.

16 - LARZILLIERE I \& BEAU P. Chronic inflammatory bowel disease and pregnancy. Case control study. Gastroenterol Clin Biol 22: 1056-1060,1998.

17 - FONAGER K; SORENSEN HT; OLSEN J; DAHLERUP JF \& RASMUSSEN SN. Pregnancy outcome for women with Crohn's disease: a follow-up study based on linkage between national registries. Am J Gastroenterol 93: 2426$2430,1998$.
18 - BRANDT LJ; ESTABROOK SG \& REINUS JF. Results of a survey to evaluate whether vaginal delivery and episiotomy lead to perineal involvement in women with Crohn's disease. Am J Gastroenterol 90: 1918-1922,1995.

19 - ILNYCKYJI A; BLANCHARD JF; RAWSTHORNE P \& BERNSTEIN CN. Perianal Crohn's disease and pregnancy: role of the mode of delivery. Am J Gastroenterol 94: 32743278,1999 .

20 - ROGERS RG \& KATZ VL. Course of Crohn's disease during pregnancy and its effect on pregnancy outcome: a retrospective review. Am J Perinatol 12: 262-264,1995.

21 - HAVERKAMP F; NORMANN D; KUSTER W \& ZERRES K. Pregnancy in Crohn's disease and ulcerative colitis. Maternal and fetal risks. Geburtshilfe Frauenheilkd 50: 339343,1990 .

22 - CASTIGLIONE F; PIGNATA S; MORACE F; SARUBBI A; BARATTA MA., D'AGOSTINO L \& D'ARIENZO A. Effect of pregnancy on the clinical course of a cohort of women with inflammatory bowel disease. Ital J Gastroenterol 28: 199204,1996.

23 - PORTO JAF \& FRAGA FILHO C. Gastroenteropatias, hepatopatias, colecistopatias. In: REZENDE J, ed. Obstetrícia, $8^{\text {th }}$ ed, Editora Guanabara Koogan, Rio de Janeiro,p. 353-357, 1998.

24 - SHOENUT JP; SEMELKA RC; SILVERMAN R; YAFFE CS \& MICFLIKIER AB. MRI in the diagnosis of Crohn's disease in two pregnant women. J Clin Gastroenterol 17: 244247,1993.

25 - RAIMUND JM; JUNG-CHAIGNEAU E; CHAMOUARD P; WITTERSHEIM C; DUCLOS B \& BAUMANN R. Diagnostic value of high resolution sonography in Crohns's disease and ulcerative colitis. Gastroenterol Clin Biol 23: 740-746,1999.

26 - GASCHE C; MOSER G; TURETSCHEK K; SCHOBER E; MOESCHL P \& OBERHUBER G. Transabdominal bowel sonography for the detection of intestinal complications in Crohn's disease. Gut 44: 112-117,1999.

27 - TEAHON K; PEARSON M; LEVIAJ \& BJARNASON I. Elemental diet in the management of Crohn's disease during pregnancy. Gut 32: 1079-1081,1991.

28 - SANDS BE. Therapy of inflammatory bowel disease. Gastroenterology 118: S68 - S82,2000.

29 - CONNELL W \& MILLER A. Treating inflammatory bowel disease during pregnancy: risks and safety of drugs therapy. Drug Saf 21: 456,1999.

30 - HABAL FM; HUI G \& GREENBERG GR. Oral 5-aminosalicylic acid for inflammatory bowel disease in pregnancy: safety and clinical course. Gastroenterology 105: 1057$1060,1993$.

31 - TENNENBAUM R; MARTEAU P; ELEFANT E; RAMBAUD JC; MODIGLIANI R; GENDRE JP \& COSNES J. Pregnancy outcome in inflammatory bowel disease. Gastroenterol Clin Biol 23: 464-469,1999.

32 - HILL J; CLARKA \& SCOTT NA. Surgical treatment of acute manifestations of Crohn's disease during pregnancy. J R Soc Med 90: 64-66,1997.

Recebido para publicação em 18/09/2000

Aprovado para publicação em 12/02/2001 